\title{
Bayesian Zero- Inflated Poisson model for prognosis of demographic factors associated with using crystal meth in Tehran population
}

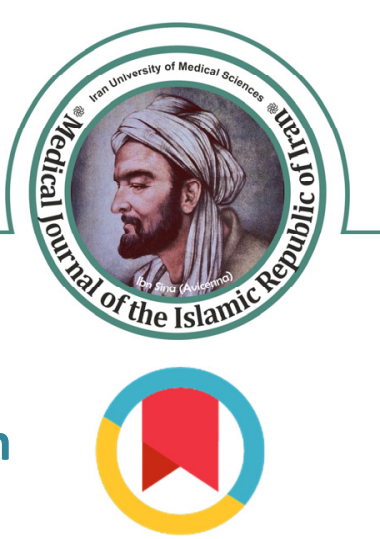

\author{
Asma Pourhoseingholi ${ }^{1,2}$, Ahmad Reza Baghestani ${ }^{1}$, Erfan Ghasemi ${ }^{1}$, Alireza Akbarzadeh Baghban ${ }^{3 *}$, Mariet Ghazarian ${ }^{4}$
}

Received: 16 Aug 2017

Published: 19 Mar 2018

\section{Abstract}

Background: Use of methamphetamine (MA) and other stimulants has increased steadily over the past 10 years. Risk factor evaluation to reduce the problem in the community is one solution to protect people from addiction. This study aimed at using Bayesian zeroinflated Poisson (ZIP) model to investigate the relationship between the number of using crystal meth and some demographic factors in Tehran population.

Methods: A cross-sectional study was conducted to investigate crystal meth abuse in Tehran, the capital of Iran, in 2012. Stratified sampling method was used to select samples from 22 urban areas of Tehran. Trained researchers referred to the public places, such as streets, parks, squares, and libraries, to perform face-to-face interviews with the randomly selected samples. Bayesian ZIP model was used to perform the analysis, and SAS 9.3 program was used for data analysis.

Results: A total of 993 individuals were studied. According to Bayesian ZIP model, sex (mean $=-0.27,95 \%$ CI $(-0.485,-0.061)$ ), age $($ mean $=0.03,95 \% \mathrm{CI}(0.018,0.043))$, high school level education (mean=1.276, 95\% CI $(0.699,01.9))$, diploma level education (mean= $10.4,95 \% \mathrm{CI}(0.511,1.69))$, and university level education (mean $=0.69,95 \% \mathrm{CI}(0.142,1.33)$ ) were all found to have significant associations with crystal meth usage, being the dependent variable.

Conclusion: Males, those with higher education levels, and older people in Tehran population are more likely to use crystal meth. This demographic information may be useful in designing preventive programs. Moreover, it is better to analyze count data with excessive zeroes using Bayesian zero- inflated model instead of the usual count models.

Keywords: Bayesian analysis, Crystal meth, Zero-inflated Poisson, Tehran population

Copyright@ Iran University of Medical Sciences

Cite this article as: Pourhoseingholi A, Baghestani AR, Ghasemi E, Akbarzadeh Baghban A, Ghazarian M. Bayesian Zero- Inflated Poisson model for prognosis of demographic factors associated with using crystal meth in Tehran population. Med J Islam Repub Iran. 2018(19 Mar);32:24. https://doi.org/10.14196/mjiri.32.24

\section{Introduction}

Opiates use has a long history in Iran (1). Especially, opium and hashish have been used since 300 years ago (2). On the other hand, Iranians have used opium and its pharmacological and psychotropic effects to manage some medical conditions related to pain $(3,4)$. However, in this century, many different abuse drugs exist, which have been

Corresponding author: Dr Alireza Akbarzadeh Baghban,akbarzad@sbmu.ac.ir

1. Department of Biostatistics, Faculty of Paramedical Sciences, Shahid Beheshti University of Medical Sciences, Tehran, Iran.

2. Department of Biostatistics, Faculty of Paramedical Sciences, Students' Research

Committee, Shahid Beheshti University of Medical Sciences, Tehran, Iran.

3. Proteomics Research Center, School of Rehabilitation, Shahid Beheshti University of Medical Sciences, Tehran, Iran.

4. Deputy of Drug Prevention and Treatment, State Welfare Organization, Tehran, Iran.

surging in Iran $(5,6)$. These different drugs encompass heroin, high-grade crystal heroin (Kerack), crystal methamphetamine, buprenorphine, cocaine, tramadol, and ecstasy $(6,7)$. Among these drugs, the use of methamphetamine (MA) has increased, especially among young Iranians (8). Recent studies have shown a rapid increase in MA usage

$\uparrow$ What is "already known" in this topic:

Recently, the use of methamphetamine (MA) has been increased in Iran, especially among the young. The smokable form of methamphetamine hydrochloride is called "crystal meth" and is more likely to cause addiction than other forms of methamphetamine. However, no study has been conducted about this drug and its risk factors in Iran.

\section{$\rightarrow$ What this article adds:}

We used Bayesian zero- inflated method to investigate the risk factors of using crystal meth in Tehran population. Bayesian approach is a good method in statistical inferences, but it has rarely been used to analyze such data. We have found an important role of some demographic factors on using crystal meth in Tehran population using this model. 
among young adults (8). For example, a study found that the mean drug abuse initiation age of $25 \%$ of substance abusers, who reported using MA, was 18 years (9). Another study revealed that $16 \%$ of addicts, who referred to addiction treatment centers, had tried MA (10). Thus, it seems that using MA and other stimulants has increased steadily over the past 10 years.

Smoking, sniffing, injection, and ingestion are the main routes of methamphetamine usage (11). However, smoking is the most common route of methamphetamine administration in Iran. The smokable form of methamphetamine hydrochloride is called "crystal meth" and is more likely to cause addiction than other forms of methamphetamine (12, 13). Many social, legal, and emotional problems are caused as the result of the increasing increase in the usage of this drug. Furthermore, the destructive effect of this drug on health makes it an important health concern in any society. Memory loss, malnutrition, aggression, psychotic behavior, and severe dental problems are the most common health problems associated with using crystal meth (14, 15). Transmission of infectious diseases, such as hepatitis and $\mathrm{HIV}$, is another major problem associated with crystal meth usage.

It seems that we can find numerous reasons for prevalence of crystal meth due to geographical location of Iran and some other reasons such as easy access, ease of production, lack of awareness of negative consequences, and lack of serious preventive programs (14). Also, there is a wrong perception about this drug in the society; for example, its effect on losing weight or helping increase wakefulness and performance $(16,17)$. Despite all that, there is limited information about this drug. As a result, it is of paramount importance to study the risk factors and other prognosis of this drug to provide more information for policymakers and set preventive programs.

It seems that demographic factors have an important role as risk factors in addiction. In medical studies, statistical modeling is used as an approach for risk factor analysis, and in this approach, selecting an appropriate model is more important. When the response variable is counted, several models could be used for data analysis. Sometimes, count data have an overdispersion problem because of having large number of zeros. This phenomenon is called zero inflation. Using usual count models in zero-inflated data causes misleading results. Lambert(18) proposed zero-inflated Poisson (ZIP) regression model for independent count data. Using these models has recently increased in the application areas of medical and health care fields (19-24). Usually, statistical inference is based on classical approaches, such as expectation-maximization (see, eg,(25)) on maximum likelihood. However, Bayesian inference is another approach for estimating the parameters. Use of Bayesian approach has recently been increased in applied statistical methods (26). Developing computational Bayesian statistics is the key point to make major changes in statistics. Bayesian estimate of zero- inflated models have been introduced just recently. Using these models instead of classical models has also been increased in application field. Accordingly, this study aimed at applying Bayesian method to estimate the parameters in a zero-inflated Poisson model. In this model, the relationship between the number of crystal meth use and some demographic factors was investigated.

\section{Methods}

\section{Participant}

The data of this study were part of a cross-sectional study that investigated crystal meth abuse in Tehran, Iran, in 2012. The sample size was selected according to previous prevalence of crystal meth in Iran (prevalence of almost $5 \%$, and $\alpha=0.05$ for calculating the sample size). The sample size formula was used according to prevalence. Those aged 14 to 55 years were selected as a target population because a previous study found this age range to be a highrisk group (9).

Stratified sampling method was conducted to select samples from 22 urban areas of Tehran. The sample size in each area was selected proportionately to the population size. Trained researchers referred to the public places, such as streets, parks, squares, and libraries, to perform face-to-face interviews with the randomly selected samples. A valid questionnaire with 62 items was used to collect data. The Cronbach alpha of this questionnaire for this study was $87 \%$, which is at an acceptable range, as it is greater than the threshold level of $70 \%$. The present study used the following demographic information: sex, age, education level (illiterate, high school, diploma, and university level education), and marital status (single, married, and widowed) as covariates and the count of using the drug as a dependent variable. Other data were collected using the same questionnaire; however, it was decided to only use the data about the count of using crystal meth and some demographic aspects of participants for this study. Informed consent was obtained from all study participants before the project began.

\section{Statistical analysis}

Descriptive statistics and frequency distribution, such as mean, standard deviation, and percentage, were calculated according to standard methods. Non- parametric tests including Mann-Whitney and Kruskal Wallis were used for univariate analysis due to non-normality of the data.

The outcome variable was the count of using crystal meth, and count models were needed to perform the analysis(27). Bayesian ZIP model was used to analyze the count data. It has been established that prior probability for all favorite parameters is needed in all Bayesian methods. In ZIP model, in addition to covariate parameters, there is a mixture parameter, for which there is a need to assume prior distribution. In this study, the mixture proportion had a uniform $(0,1)$ prior distribution, and all covariates parameters had diffuse normal $\left(0 . \sigma^{2}=1000\right)$ prior distribution, expressing the lack of knowledge about the regression parameters. P-values less than 5\% were considered as significant. For categorical variables, $p$-values were compared with reference group. Because of using Bayesian approach in the analysis, the credit interval was used to make a decision about the significant variables. When the credit interval included zero, the parameter was considered not significant. 
Markov chain Mont Carlo (MCMC) algorithm was employed for fitting the Bayesian model. The SAS 9.3 university edition (free statistical software) program was used to compute the results.

\section{Results}

A total of 993 individuals were investigated in this study, among whom 448 (45.1\%) were female. The mean (SD) age was 25.98 (5.96) years, ranging from 14 to 52 years. Table 1 demonstrates the distribution of different characteristics of the participants.

Of the entire participants in this study, 74 persons (7.5\%) have reported at least one time use of crystal meth in their life. Distribution of the number of crystal usage among the studied participants is presented in Table 2. The number of using crystal meth was found to be statistically different between male and female participants $(\mathrm{p}<0.001)$. Also, educational level and marital status were found to be significant predictors of using crystal $(\mathrm{p}<0.001)$. The mean of using crystal in males was higher than in females; this mean was higher in the widowed and those with high school level education (Table 3 ).

In the next step of this study analysis, Bayesian ZIP was used to investigate the effect of all considered factors on the number of using crystal meth simultaneously. Table 4 demonstrates the result of this model. ZIP model revealed that sex, age, high school level education, diploma level education, and university level education were associated with using crystal meth. According to these results, females $($ mean $=-0.27$; 95\% CI $(-0.485,-0.061))$, age $($ mean $=0.03$; $95 \% \mathrm{CI}(0.018,0.043)$, high school level education (mean=

Table 1. Distribution of demographic factors among the population of this study

\begin{tabular}{lccc}
\hline Variable & Category & $\mathrm{n}$ & $\%$ \\
\hline Sex & Men & 545 & 54.9 \\
& Women & 448 & 45.1 \\
Marital status & Single & 602 & 61.1 \\
& Married & 366 & 37.1 \\
\multirow{4}{*}{ Education } & Widowed & 18 & 1.8 \\
& Illiterate & 16 & 1.6 \\
& High school & 224 & 22.9 \\
& Diploma & 544 & 55.6 \\
& University & 194 & 19.8 \\
\hline
\end{tabular}

Table 2. Distribution of number of using crystal meth among the population of this study

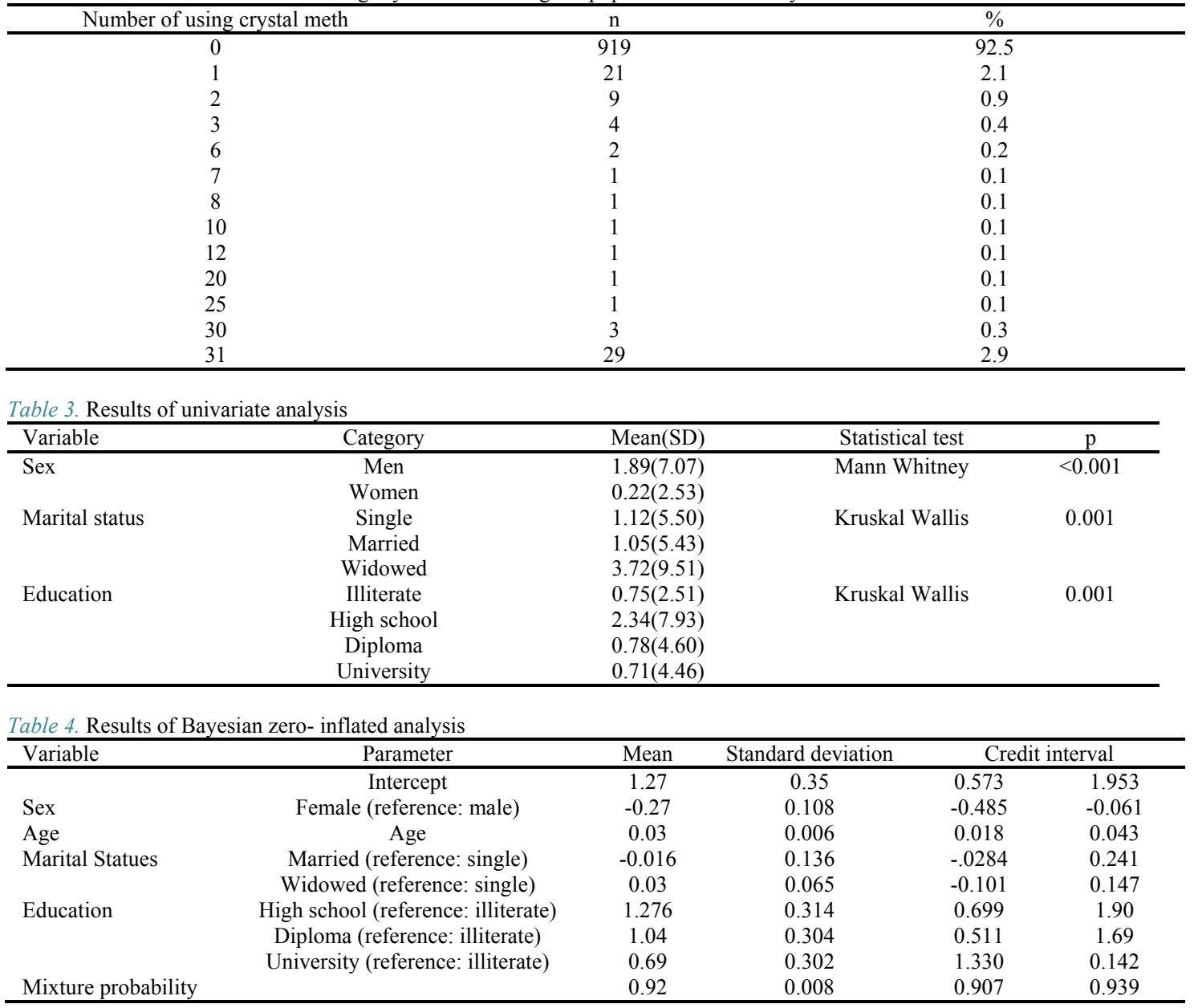


1.276 ; 95\%CI $(0.699,1.90))$, diploma level education $($ mean $=1.04 ; 95 \% \mathrm{CI}(0.511,1.69))$, and university level education level of education (mean $=0.69 ; 95 \%$ CI $(0.142$, 1.330)) were found to have significant association with crystal usage. The expected mean of the frequency of using crystal meth in females, younger people, and illiterates were less than in males, older people, and those with higher education, respectively.

\section{Discussion}

According to this study, the prevalence of at least one time use of crystal meth in Iranian population was $7.5 \%$. The information regarding this drug among the population in general is limited. According to Drug Situation Analysis Report of the United Nation Office of Drug and Crime, methamphetamine usage has increased from none in 2004 to $3.5 \%$ in 2007 in Iran (28), so it seems there is a dramatic change in this prevalence during 5 years. On the other hand, due to lack of data in many countries, there is no report on its exact worldwide prevalence. However, there are several reports from other countries who reported the prevalence of meth including Philippines (10\%), Guam (7\%), Estonia (5.5\%), Nicaragua and New Zealand (4\%), Poland (3.7\%), Denmark (3.1\%), Germany $(2.4 \%)$, and France $(1.8 \%)$. It seems that the prevalence may have increased in recent years (29).

In this study, sex, age, and education had a statistically significant relationship with crystal meth usage in the studied population in Tehran. According to the results of this study, the expected mean of the frequency of using crystal meth is almost $25 \%$ less in females. Also, the expected mean among people with high school level education, diploma level education, and university level education was $3.58,2.82$, and 1.99 times more than illiterate people, respectively. In addition, older people were more likely to use crystal meth, and the expected mean was 1.03 time of the younger people. In another study done in Hamedan (an Iranian province in the west), being single and having higher education level had a significant relationship with using crystal meth (8). Another study on Iranian heroin kerackdependent females revealed that younger and low- educated people in that population were more likely to use methamphetamine (30). This study and also the Hamedan study both indicated that highly educated people were a group atrisk However, another study on heroin kerack-dependent females had completely different results, showing that the population of the study is important.

As mentioned earlier, there are limited studies on crystal meth usage, so comparing other results with this study is not possible. In contrast, this can show the importance of this study being among the first of its kind. Lack of studies and information about using this drug can affect preventive strategies. Furthermore, a long questionnaire that we used for collecting information in this study may have affected the participants and could be one weak point of this study.

The main feature of the data of this study was count. The first count model that researchers referred to was Poisson regression (PR) model. However, because of the related problem of this model (overdispersion), sometimes this model is not suitable and its results cannot be trusted. According to the source of overdispersion, using remedial techniques is needed. Sometimes, the main reason for this overdispersion is due to many zeroes in the data set. In this case, there are different methods for excessive zero data, such as zero-inflated models, which were used in this paper and in Hurdle models (31). Many studies have applied these models recently (32-37). There are 2 different approaches to estimate the parameters in statistical modeling there are two different approaches: classical and Bayesian approach. Classical methods include different methods, such as maximum likelihood estimation (MLE), which has some drawbacks. Uncertainty in the parameter estimates is one of the problems. Also, these methods are usually based on the asymptotic normality assumption, which is often not tenable for small sample (sparse) data $(38,39)$. However, Bayesian approach corrects these drawbacks of the classical estimation; specially, the estimation according to Bayesian method are reliable regardless of the smallness of the data (40). On the other hand, recent advances in simulation method, such as Markov chain Monte Carlo calculation, now allow for fitting models of this type, which have a complex structure (41). In recent years, several authors have proposed Bayesian alternatives to fitting zero-inflated models $(42,43)$. In the application field, this method also has been used by different authors in different research areas.

Jang et al. have considered zero-inflated Poisson and zero-inflated negative binomial regression models to analyze discrete count road safety data. They estimated regression coefficients associated with several safety countermeasures using Bayesian method. Their empirical results showed that zero-inflated models perform better than the classical approach (44).

In a study investigating spatiotemporal HIV/TB child mortality, Bayesian analysis of zero inflation was used by Musenge et al. They used a computationally efficient alternative to Markov chain Monte Carlo (MCMC) called 'Integrated Nested Laplace Approximation' to estimate the parameters (45).

In another HIV mortality study, Musal and Aktekin introduced parameter estimation issues of Bayesian zero-inflated Poisson models and discussed MCMC method implications(46).

Swallow et al. have considered ecological data corresponding to an annual average of 26 weekly maximum counts of birds. They used Bayesian hierarchical Tweedie regression model that can directly accommodate the excess number of zeroes common to this type of data. Implementation of the model is conducted in a Markov chain Monte Carlo (MCMC) framework (47).

\section{Conclusion}

Thus, it seems that using these Bayesian models have increased in recent years. In this study, Bayesian ZIP model was performed to examine related demographic factors with count of using crystal meth. In conclusion, using Bayesian approach because of its benefits, compared to classical approach, is a good alternative for statistical modeling in different fields of studies. Another important issue 
of this study was that to analyze count data with zeroes, needs arise for conducting the analysis adopting special zero- inflated modeling approach instead of the usual count modeling. Therefore, it is essential to apply the appropriate count model (zero- inflated in this case) to have meaningful results.

\section{Ethical considerations}

Ethical issues (plagiarism, informed consent, misconduct, data fabrication and/or falsification, double publication and/or submission, redundancy, etc.) have been completely adhered to by the authors of this study.

\section{Acknowledgement}

This paper was part of a research for a $\mathrm{PhD}$ degree at School of Paramedical Sciences, Shahid Beheshti University of Medical Sciences (BUMS) and was financially supported by the mentioned university.

\section{Conflict of Interests}

The authors declare that they have no competing interests.

\section{References}

1. Mokri A. Brief overview of the status of drug abuse in Iran. Arch Iran Med. 2002;5(3):184-90.

2. Day C, Nassirimanesh B, Shakeshaft A, Dolan K. Patterns of drug use among a sample of drug users and injecting drug users attending a General Practice in Iran. Harm Reduct J. 2006;3:2.

3. Jafari S, Rahimi-Movaghar A, Craib K, Baharlou S, Mathias R. Sociocultural Factors Associated with the Initiation of Opium Use in Darab, Iran. Int J Ment Health Addict. 2009;7(2):376-88.

4. Karrari P, Mehrpour O, Balali-Mood M. Iranian Crystal: A misunderstanding of the crystal-meth. J Res Med Sci. 2012;17(2):2034.

5. Mehrpour O. Methamphetamin abuse a new concern in Iran. Daru. 2012;20(1):73.

6. Momtazi S, Rawson R. Substance abuse among Iranian high school students. Curr Opin Psychiatry. 2010;23(3):221-6.

7. Zamani S, Farnia M, Tavakoli S, Gholizadeh M, Nazari M, Seddighi $\mathrm{AA}$, et al. A qualitative inquiry into methadone maintenance treatment for opioid-dependent prisoners in Tehran, Iran. Int J Drug Policy. 2010;21(3):167-72.

8. Barati M, Ahmadpanah M, Soltanian AR. Prevalence and factors associated with methamphetamine use among adult substance abusers. J Res Health Sci. 2014;14(3):221-6.

9. Narenjiha H, Rafiey AH, Baghestani AR, Noori R, Ghafori B, Soleimani L. Rapid situation assessment of substance abuse and dependency in Islamic Republic of Iran. Tehran, IR: Danzheh Publisher; 2008.

10. Ahmadpanah M, Mirzaei Alavijeh M, Allahverdipour H, Jalilian F, Haghighi M, Afsar A, et al. Effectiveness of Coping Skills Education Program to Reduce Craving Beliefs among Addicts Referred To Addiction Centers in Hamadan: A Randomized Controlled Trial. Iran J Public Health. 2013;42(10):1139-44.

11. Vocci FJ, Appel NM. Approaches to the development of medications for the treatment of methamphetamine dependence. Addiction. 2007;102 Suppl 1:96-106.

12. Jafari S, Rahimi-Movaghar A, Craib KJP, Baharlou S, Mathias R. A follow-up study of drug users in Southern Iran. Addict Res Theory. 2010;18(1):59-70.

13. Afsharnezhad S, Mahmoodi M, Erfanian A, Pormahmood J, Jamialahmadi K. Studying the increase of activity level of serum alkaline phosphate in crystal addicts. J Med Sci Islam Azad Univ Mashhad. 2009;5(2):85-90.

14. Barati M, Allahverdipour H, Moinei B, Farhadinasab A, Mahjub H. Evaluation of theory of planned behavior-based education in prevention of MDMA (ecstasy) use among university students. Med J Tabriz Univ
Med Sci. 2011;33(3):20-9

15. Iritani BJ, Hallfors DD, Bauer DJ. Crystal methamphetamine use among young adults in the USA. Addiction. 2007;102(7):1102-13.

16. Bashirian S, Hidarnia A, Allahverdipour H, Hajizadeh E. Application of the theory of planned behavior to predict drug abuse related behaviors among adolescents. J Res Health Sci. 2012;12(1):54-60.

17. Barati M, Allahverdipour H, Moeini B, Farhadi Nasab A, Mahjub H, Jalilian F. Assertiveness Skills Training Efficiency on College Students' Persuasive Subjective Norms against Substance Abuse. Sci J Hamadan Univ Med Sci. 2011;18(3):40-9.

18. Lambert D. Zero-inflated Poisson regression, with an application to defects in manufacturing. Technometrics. 1992;34(1):1-14.

19. Baughman AL. Mixture model framework facilitates understanding of zero-inflated and hurdle models for count data. J Biopharm Stat. 2007;17(5):943-6.

20. Gilthorpe MS, Frydenberg M, Cheng Y, Baelum V. Modelling count data with excessive zeros: the need for class prediction in zero-inflated models and the issue of data generation in choosing between zeroinflated and generic mixture models for dental caries data. Stat Med. 2009;28(28):3539-53.

21. Picard RR, Cook RD. Cross-Validation of Regression Models. J Am Stat Assoc. 1984;79(387):575-83.

22. Gardner W, Mulvey EP, Shaw EC. Regression analyses of counts and rates: Poisson, overdispersed Poisson, and negative binomial models. Psychol Bull. 1995;118(3):392-404.

23. Hardin JW, Hilbe J. Generalized Linear Models and Extensions. Texas: StataCorp LP; 2012.

24. Mullahy J. Specification and testing of some modified count data models. J Econom. 1986;33(3):341-65.

25. Ruli E, Ventura L. Modern Bayesian Inference in Zero-Inflated Poisson Models. 46th Scientific Meeting of the Italian Statistical Society. 2012;2012.

26. Bolstad WM. Wiley Series in Computational Statistics. Understanding Computational Bayesian Statistics: John Wiley \& Sons, Inc.; 2009.

27. Hedeker D, Gibbons RD. Longitudinal Data Analysis. New Jersey: NY: John Wiley \& Sons; 2006.

28. Degenhardt L, Mathers B, Guarinieri M, Panda S, Phillips B, Strathdee S, et al. The Global Epidemiology of Methamphetamine Injection: A Review of the Evidence on Use and Associations with HIV and Other Harm. Sydney, Australia: National Drug \& Alcohol Research Centre; 2007.

29. Drug Situation Analysis Report Islamic Republic of Iran. Vienna; 2010.

30. Alam Mehrjerdi Z, Abarashi Z, Mansoori S, Deylamizadeh A, Salehi Fadardi J, Noroozi A, et al. Methamphetamine Use Among Iranian Heroin Kerack-Dependent Women: Implications for Treatment. Int $\mathrm{J}$ High Risk Behav Addict. 2013;2(1):15-21.

31. Barondess DA, Meyer EM, Boinapally PM, Fairman B, Anthony JC. Epidemiological evidence on count processes in the formation of tobacco dependence. Nicotine Tob Res. 2010;12(7):734-41.

32. Dwivedi AK, Dwivedi SN, Deo S, Shukla R, Kopras E. Statistical models for predicting number of involved nodes in breast cancer patients. Health. 2010;2(7):641-51.

33. Bergemann TL, Huang Z. A new method to account for missing data in case-parent triad studies. Hum Hered. 2009;68(4):268-77.

34. Carrel M, Escamilla V, Messina J, Giebultowicz S, Winston J, Yunus $\mathrm{M}$, et al. Diarrheal disease risk in rural Bangladesh decreases as tubewell density increases: a zero-inflated and geographically weighted analysis. Int J Health Geogr. 2011;10(1):41.

35. Carrel M, Voss P, Streatfield PK, Yunus M, Emch M. Protection from annual flooding is correlated with increased cholera prevalence in Bangladesh: a zero-inflated regression analysis. Environ Health. 2010;9:13.

36. Abadi A, Pourhoseingholi A, Chaibakhsh S, Safaee A, MoghimiDehkordi B. Specific count model for investing the related factors of cost of GERD and functional dyspepsia. Gastroenterol Hepatol Bed Bench. 2013;6(Suppl 1):S122-8.

37. Akbarzadeh Baghban A, Pourhoseingholi A, Zayeri F, Jafari AA, Alavian SM. Application of zero-inflated poisson mixed models in prognostic factors of hepatitis C. Biomed Res Int. 2013;2013:403151.

38. Gill J. Bayesian Methods: A Social and Behavioral Sciences Approach. New York, NY: Chapman \& Hall/CRC; 2002.

39. Gelman A, Carlin J, Stern H, Rubin DB. Bayesian Data Analysis. New York, NY: Chapman \& Hall; 1995. 
40. Agresti A. An Introduction to Categorical Data Analysis. New York, NY; 1996.

41. Gilks WR, Richardson S, Spiegelhalter DJ. Markov Chain Monte Carlo in Practice. London, UK: Chapman \& Hall; 1996.

42. Rodrigues J. Bayesian Analysis of Zero-Inflated Distributions. Commun Stat Theory Methods. 2003;32(2):281-9.

43. Ghosh SK, Mukhopadhyay P, Lu J-C. Bayesian analysis of zeroinflated regression models. J Stat Plan Inference. 2006;136(4):1360-75.

44. Jang H, Lee S, Kim SW. Bayesian analysis for zero-inflated regression models with the power prior: applications to road safety countermeasures. Accid Anal Prev. 2010;42(2):540-7.

45. Musenge E, Chirwa TF, Kahn K, Vounatsou P. Bayesian analysis of zero inflated spatiotemporal HIV/TB child mortality data through the INLA and SPDE approaches: Applied to data observed between 1992 and 2010 in rural North East South Africa. Int J Appl Earth Obs Geoinf. 2013;22(100):86-98.

46. Musal M, Aktekin T. Bayesian spatial modeling of HIV mortality via zero-inflated Poisson models. Stat Med. 2013;32(2):267-81.

47. Swallow B, Buckland ST, King R, Toms MP. Bayesian hierarchical modelling of continuous non-negative longitudinal data with a spike at zero: An application to a study of birds visiting gardens in winter. Biom J. 2015. 